\title{
An Unusual Case of Sternoclavicular Joint Infection and Lung Abscess
}

\section{Carina Rôlo Silvestre1, Ricardo Cordeiro1, Daniel Duarte1, Hugo Ferreira², Carla Cardoso1, Teresa Falcão1, António Domingos' ${ }^{1}$}

${ }^{1}$ Pulmonology Department, Centro Hospitalar do Oeste, Torres Vedras, Portugal

${ }^{2}$ Cardiothoracic Surgery Department, Centro Hospitalar Lisboa Norte, Lisboa, Portugal

Email: carinarolosilvestre@gmail.com

How to cite this paper: Silvestre, C.R., Cordeiro, R., Duarte, D., Ferreira, H., Cardoso, C., Falcão, T. and Domingos, A. (2020) An Unusual Case of Sternoclavicular Joint Infection and Lung Abscess. Open Journal of Respiratory Diseases, 10, 43-48. https://doi.org/10.4236/ojrd.2020.102005

Received: January 12, 2020

Accepted: May 4, 2020

Published: May 7, 2020

Copyright $\odot 2020$ by author(s) and Scientific Research Publishing Inc. This work is licensed under the Creative Commons Attribution International License (CC BY 4.0).

http://creativecommons.org/licenses/by/4.0/

\begin{abstract}
Septic arthritis of the sternoclavicular joint is a rare and serious infection. A delay in the diagnosis may lead to serious complications. The appropriate medical and surgical treatment is crucial to the outcome. This article aims to report our experience in the successful management of sternoclavicular joint infection complicated with a lung infection. The authors present an unusual case of sternoclavicular joint infection extending into lung parenchyma with lung abscess formation in a diabetic patient, with uncontrolled diabetes that was successfully treated. At revaluation, he was asymptomatic with no evidence of relapse. Although sternoclavicular joint infection is a rare condition we highlight the importance of clinician's awareness for an early diagnosis and treatment with broad-spectrum antibiotics and surgery.
\end{abstract}

\section{Keywords}

Lung Abscess, Septic Arthritis, Sternoclavicular Joint, Diabetes Mellitus

\section{Introduction}

Infectious diseases are more prevalent and severe in patients with diabetes mellitus, which increases their morbidity. Poor glycemic control favors immune dysfunction [1].

Septic arthritis of the sternoclavicular joint (SCJ) is an infection of the joint where the medial head of the clavicle attaches to the manubrium of the sternum and the cartilage of the first rib. SCJ is the only bony articulation between the upper extremity and the axial skeleton. It's a rare and serious infection, with less than 250 cases reported in the literature over the past five decades. It accounts 
for less $1 \%$ of all joint infections, but this increases to $17 \%$ in intravenous drug users [2] [3] [4]. The risk factors for SCJ infection include trauma, intravenous drug abuse, infected central venous catheters, intraarticular injections, rheumatoid arthritis, diabetes mellitus, human immunodeficiency virus infection, alcoholism, chemotherapy and long term use of steroids. However, some cases have been reported in healthy patients [2] [5] [6].

A delay in the diagnosis may lead to serious complications such as empyema, osteomyelitis, mediastinitis or abscess formation [3] [5]. Because of its rarity, there are no standardized treatment recommendations, so the management of SCJ infections can be very challenging and besides medical treatment a surgical approach may involve thoracic and plastic surgeons [4] [5] [7]. Recent studies recommend that all symptomatic SCJ infections require surgical intervention [8] [9].

Staphylococcus aureus is the major microbiologic agent found in SCJ infections, followed by Pseudomonas aeruginosa [2] [3]. Broad-spectrum antibiotics covering methicillin-resistant Staphylococcus aureus are recommended [8]. The duration of antibiotic treatment ranges from 4 to 6 weeks [9].

\section{Case Report}

The authors present a case of a 65-year-old male patient, ex-smoker (30 pack-years), with dental caries and history of arterial hypertension and poorly controlled diabetes mellitus, medicated with long-acting insulin and metformin. The patient presented with a four-week history of mild productive cough and left anterior chest pain that irradiated to the left shoulder. Two weeks later he noted a swelling of the left clavicular region and pain with motion of the left shoulder. The patient denied fever, chills, night sweats or weight loss. No history of alcohol, intravenous drug abuse, immunosuppressive status or trauma.

At physical examination the patient was normotensive $(125 / 65 \mathrm{mmHg})$, with a normal heart rate $(86$ beats $/ \mathrm{min})$, febrile $\left(38.3^{\circ} \mathrm{C}\right)$, with a respiratory rate of 18 breaths/min, with oxygen saturation superior to $95 \%$ on room air, and presenting a visible and palpable warm and tender mass, measuring $7 \mathrm{~cm}$, located at the left SCJ, without erythema. Pulmonary auscultation revealed inspiratory rales at the upper zone of the left hemithorax. He had no other abnormal findings.

The laboratory tests showed leukocytosis (white blood cell count 17.800 cells $/ \mu \mathrm{L} ; 86.8 \%$ neutrophils), an elevated C-reactive protein $(11.2 \mathrm{mg} / \mathrm{dL})$, plate-

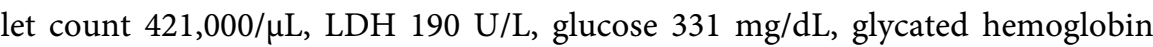
$11 \%$, blood urea nitrogen $44 \mathrm{mg} / \mathrm{dL}$ and creatinine $0.87 \mathrm{mg} / \mathrm{dL}$. Spot urine test revealed proteinuria (urine protein $1+$ ) and glycosuria (urine sugar $4+$ ). Blood cultures were negative. Rheumatologic serologies were negative.

Chest $\mathrm{x}$-ray showed at the left paratracheal zone around hyperlucent image.

Computed tomography (CT) of the chest revealed bone destruction of the left 
SCJ, involving both bones, the manubrium and clavicle, containing air, a subcutaneous air bubble measuring $7.5 \times 4 \mathrm{~cm}$ adjacent to the SCJ and at the upper left lobe a pulmonary cavitated lesion measuring $4.4 \times 5.5 \mathrm{~cm}$ that had a $10 \mathrm{~mm}$ communication between them without mediastinal or axillary adenopathy's Figure 1.

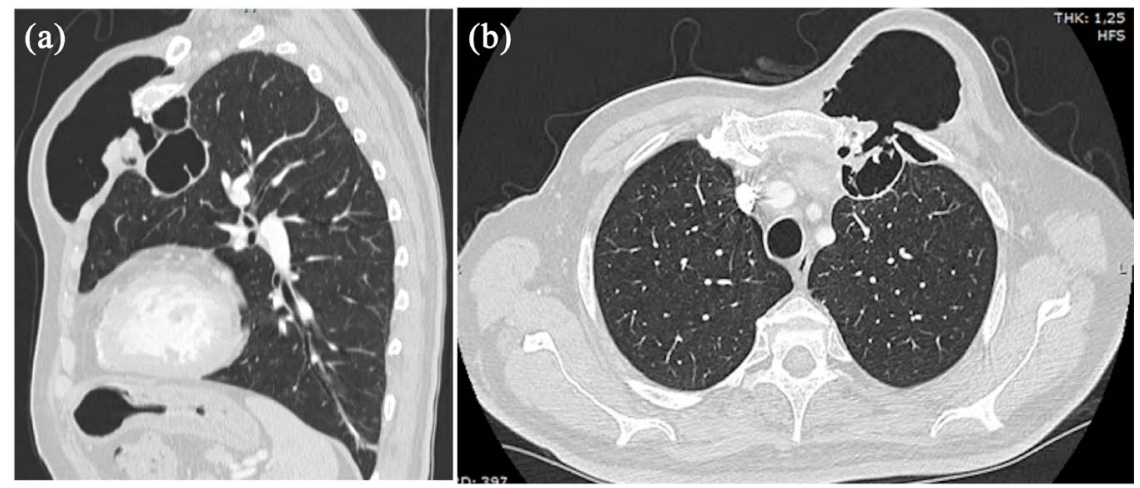

Figure 1. Chest computed tomography before surgical treatment. (a) shows at the anterior thoracic wall a subcutaneous air bubble bellow is visible bone destruction of the left SCJ and a cavitated lesion in the adjacent lung parenchyma. (b) shows a communication between the subcutaneous air bubble and the lung abscess and bone involvement with left SCJ destruction.

Bronchoscopy showed no abnormal findings, microbiologic and mycobacterial culture of bronchoalveolar lavage was negative.

The case was discussed with the cardiothoracic surgery department. The patient had no condition to be submitted to cutaneous drainage of the joint, because of the presence of the subcutaneous air bubble communicating with the lung.

Empiric intravenous antibiotic therapy with piperacillin/tazobactam associated with vancomycin and clindamycin was initiated, resulting in clinical and laboratorial improvement. Pain and mass size reduction was associated with cough with purulent sputum, related to spontaneous abscess drainage to the airway.

Two weeks after antibiotic treatment the patient was submitted to surgical debridement of the joint abscess under general anesthesia. The mycobacterial and anaerobic testing of samples collected, as the histological examination for malignancy, were negative. The microbiological culture revealed Pseudomonas aeruginosa.

Negative pressure wound therapy was applied and antibiotic therapy with piperacillin/tazobactam was extended for three more weeks until a second surgical multidisciplinary approach (thoracic and plastic surgeons) with resection of all the affected tissue and closure of the bronchopleural fistulae with biological glue and an ipsilateral pectoralis major muscle flap coverage.

The patient was discharged nine days after surgical treatment with a remarkable radiological and clinical improvement Figure 2. 

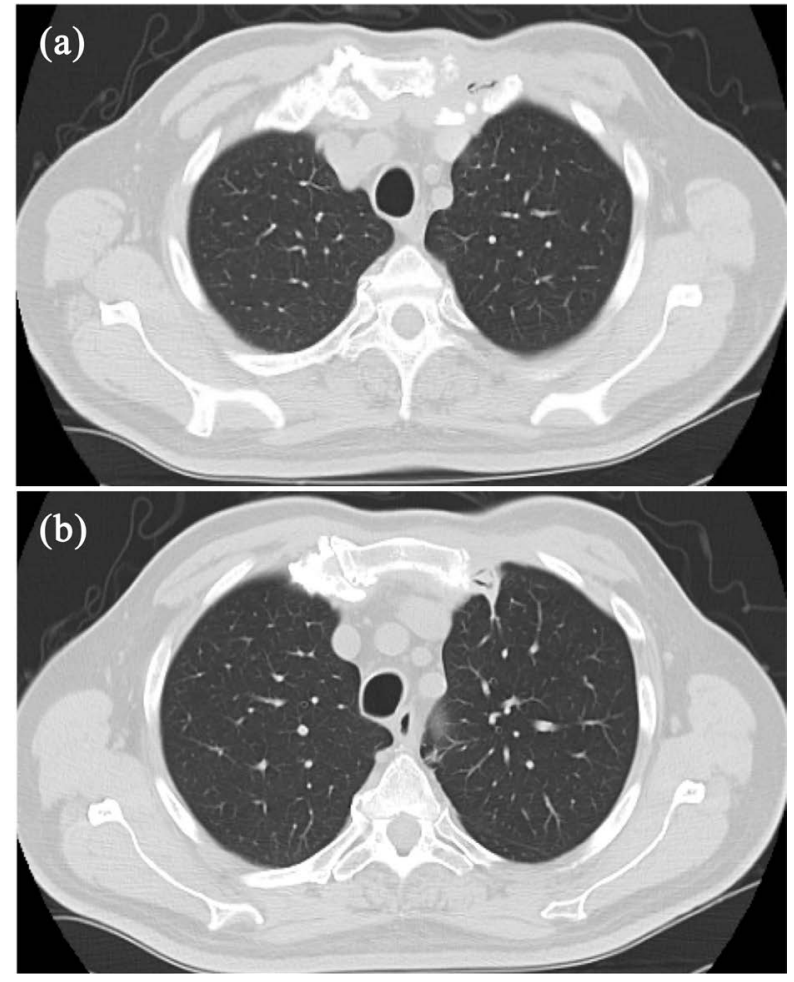

Figure 2. Two weeks after the last surgery, chest computed tomography shows complete resolution of the lung abscess, without parenchymal and soft tissue lesions. It is visible residual alterations of the left SCJ, resultant from resection of all the affected tissue, included bone.

After one-year, the patient had no recurrence and showed no disability.

\section{Discussion}

Septic arthritis of the SCJ is rare. Diabetes mellitus is the most common risk factor associated with SCJ infection, particularly, uncontrolled hyperglycemia as described in our patient [2] [3] [9].

In our patient, the chest CT finding was consistent with SCJ infection, and the SCJ infection seemed to directly extend into the left upper pulmonary lobe.

The diagnosis of septic arthritis was inferred by suggestive clinical and typical radiographic findings, persistently elevated inflammatory markers and positive microbiological culture data to Pseudomonas aeruginosa. Although Staphylococcus aureus is the most common agent found in this type of infection, Pseudomonas aeruginosa is the second most frequent microbiologic agent found [2] [3].

It was unclear whether the initial event was a bone joint infection or a lung infection/abscess, but the clinical evolution and the surgical findings allowed us to assume a primary SCJ septic arthritis, with associated lung abscess. Diabetes favors immune dysfunction, increasing susceptibility to infections, including rare ones, in this case, this was the predisposing risk factor.

Previous publications advocate broad-spectrum antibiotics and debridement of the joint as a key to a positive outcome [8] [9]. The need for muscle flap de- 
pends on the size of the deformity. In our case bone was resected as well, it seems that the patients with joint resection have better outcomes [4] [8]. Our patient had no functional impairment.

\section{Conclusion}

This case demonstrates the effect of poorly controlled Diabetes mellitus on the immune system and how exuberant and unusual infections can develop. SCJ infection is a rare condition and a potentially life-threatening because of the tight anatomic connection with the chest vascular structures. The positive outcome of this case with infection resolution and restoration of joint function highlights the importance of rapid diagnosis and prompt treatment, with a multidisciplinary approach, being broad-spectrum antibiotics and surgery crucial to the final result.

\section{Ethical Aspects}

A voluntary consent form was obtained from the patient.

\section{Conflicts of Interest}

The authors declare no conflicts of interest regarding the publication of this paper.

\section{References}

[1] Abu-Ashour, W., Twells, L., Valcour, J., et al. (2017) The Association between Diabetes Mellitus and Incident Infections: A Systematic Review and Meta-Analysis of Observational Studies. BMJ Open Diabetes Research \& Care, 5, 1-10. https://doi.org/10.1136/bmjdrc-2016-000336

[2] Khan, K., Wozniak, S.E., Mehrabi, E., Giannone, A.L. and Dave, M. (2015) Sternoclavicular Osteomyelitis in an Immunosuppressed Patient: A Case Report and Review of the Literature. American Journal of Case Reports, 16, 908-911. https://doi.org/10.12659/AJCR.895803

[3] Ross, J. and Shamsuddin, H. (2004) Sternoclavicular Septic Arthritis: Review of 180 Cases. Medicine, 83, 139-148. https://doi.org/10.1097/01.md.0000126761.83417.29

[4] Schipper, P. and Tieu, B.H. (2017) Acute Chest Wall Infections: Surgical Site Infections, Necrotizing Soft Tissue Infections, and Sternoclavicular Joint Infection. Thoracic Surgery Clinics, 27, 73-86. https://doi.org/10.1016/j.thorsurg.2017.01.001

[5] Nusselt, T., Klinger, H.M., Freche, S., Schultz, W. and Baums, M.H. (2011) Surgical Management of Sternoclavicular Septic Arthritis. Archives of Orthopaedic and Trauma Surgery, 131, 319-323. https://doi.org/10.1007/s00402-010-1178-0

[6] Henriksen, J., Tang, M. and Hjortdal, V. (2015) Abscess Formation after Septic Arthritis in the Sternoclavicular Joint of Two Healthy Men. Case Reports in Surgery, 2015, 1-2. https://doi.org/10.1155/2015/292854

[7] Muesse, J.L., Blackmon, S.H., Ellsworth, W.A. and Kim, M.P. (2014) Treatment of Sternoclavicular Joint Osteomyelitis with Debridement and Delayed Resection with Muscle Flap Coverage Improves Outcomes. Surgery Research and Practice, 2014, 1-6. https://doi.org/10.1155/2014/747315

[8] Murga, A., Copeland, H., Hargrove, R., Wallen, J.M. and Zaheer, S. (2017) Treat- 
ment for Sternoclavicular Joint Infections: A Multi-Institutional Study. Journal of Thoracic Disease 9, 1503-1508. https://doi.org/10.21037/jtd.2017.05.76

[9] von Glinski, A., Yilmaz, E., Rausch, V., Koenigshausen, M., Schildhauer, T.A., Seybold, D. and Geßmann, J. (2019) Surgical Management of Sternoclavicular Joint Septic Arthritis. Journal of Clinical Orthopaedics and Trauma, 10, 406-413. https://doi.org/10.1016/j.jcot.2018.05.001 